\title{
ESTADO NUTRICIONAL E HÁBITOS DE VIDA EM ESCOLARES
}

\author{
NUTRITIONAL STATUS AND HABITS OF LIFE IN \\ SCHOOL CHILDREN
}

\author{
Carla Campos Muniz Medeiros', Maria Aparecida Alves Cardoso', \\ Renner Augusto Raposo Pereira² Guilherme Tarso de Andrade Alves ${ }^{3}$, \\ Inacia Sátiro Xavier de França ${ }^{4}$, Alexsandro Silva Coura ${ }^{5}$ \\ Danielle Franklim de Carvalho ${ }^{6}$
}

\begin{abstract}
RESUMO
Objetivou-se descrever a prevalência de obesidade, sobrepeso, hábitos alimentares e a frequência de atividade física dos escolares da cidade de Campina Grande, Paraíba. Estudo realizado com 255 escolares (6 a 10 anos), dos quais foi calculado o IMC. Considerou-se sobrepeso IMC $\geq 85$ e $<95$, e obesidade, IMC $\geq 95$. A prevalência de sobrepeso e obesidade foi de $23,5 \%$, sendo mais frequente entre os alunos da rede privada $(49,1 \%)$, do que os da pública $(17,1 \%)$ ( $p<0,001)$, e em meninos $(31,2 \%)$ do que meninas $(16 \%)(p<0,01)$. A maioria das crianças $(66,3 \%)$ praticava menos que três dias de atividade física por semana e foi observada uma frequência maior de lanches levados de casa para escola pelos alunos das escolas privadas $(83,7 \%)$, sendo os de maior preferência: biscoito (72\%), refrigerante (54\%) e salgadinho (50\%). Já os escolares da rede pública apresentaram maior consumo de biscoito $(68,2 \%)$, iogurte $(61,9 \%)$ e salada de frutas $(55,6 \%)$. Obesidade, sobrepeso, hábitos alimentares não saudáveis e sedentarismo foram frequentes entre os escolares. Este fato reforça a necessidade de implantação de políticas públicas voltadas para a prevenção da obesidade, bem como a intensificação da educação nutricional e do estímulo à atividade física nas escolas.
\end{abstract}

Palavras-chave: obesidade; sobrepeso; criança.

Estudo vinculado a Unidade Acadêmica de Medicina. Universidade Federal de Campina Grande (UFCG). Rua Aprígio Veloso, 882, Bodocongó. Campina Grande-PB, CEP.: 58109-000.

1 Doutora. Programa de Pós-Graduação em Saúde Pública/Universidade Estadual da Paraíba (UEPB)E-mail: carlamunizmedeiros@hotmail.com

Iniciação científica, Universidade Federal de Campina Grande (UFCG)E-mail: renner_augusto@hotmail.com

Iniciação científica, Universidade Federal de Campina Grande (UFCG) E-mail: guilherme tarso@hotmail.com

4 Doutora. Bolsista de Produtividade do Conselho Nacional de Desenvolvimento Científico e Tecnológico (CNPq). Programa de Pós-Graduação em Saúde Pública/Universidade Estadual da Paraíba (UEPB). E-mail: isxf@oi.com.br

5 Mestrando. Bolsista da Coordenação de Aperfeiçoamento de Pessoal de Nível Superior (CAPES). Programa de PósGraduação em Saúde Pública/Universidade Estadual da Paraíba (UEPB). E-mail: alex@uepb.edu.br

6 Doutorado. E-mail: daniellefranklim6@gmail.com

Correspondência para: Carla Campos Muniz Medeiros. Rua Vigário Calixto, 1450, Bloco: H, apto. 103 - Catolé Campina Grande-PB, CEP: 58104-481. Tel: (83) 8859-4107. E-mail: carlamunizmedeiros@hotmail.com

Como citar este artigo: Medeiros CMM et al. Nutritional status and habits of life in school children. Journal of Human Growth and Development 2011; 21(3): 789-797.

Artigo submetido em 20.12.10, aceito em 20.08.11. 


\begin{abstract}
This study aimed to describe the prevalence of obesity, overweight, eating habits and the frequency of physical activity among schoolchildren from Campina Grande, Paraíba. It included 255 children from six to ten years old, whose BMI were calculated. It was considered overweight BMI $\geq 85$ percentile $<95$, and obesity, BMI $\geq 95$ percentile. Overweight and obesity was observed in $23.5 \%$ of the studied population and was higher in private school students $(49.1 \%)$ than those of the public $(17.1 \%)(p<0.001)$, and in the boys $(31,2 \%)$ than girls $(16 \%)(p<0.01)$. The majority of the children $(66.3 \%)$ performed less than three days of physical activity per week and was observed a higher frequency of snack brought from home to school by students of private schools $(83.7 \%)$, between them: biscuit (72\%), soft drink (54\%) and snacks (50\%). The public school students had a highest intakes of biscuits $(68.2 \%)$, yoghurt $(61.9 \%)$ and fruit salad (55.6\%). Obesity, overweight, unhealthy eating habits and sedentarism were frequent among schoolchildren. This reinforces the need to implement public policies for the prevention of obesity, as well as an intensification of nutrition education and encouraging the physical activity in schools.
\end{abstract}

Key words: obesity; overweight; child.

\section{INTRODUÇÃO}

A obesidade vem aumentando de forma alarmante, sendo considerada uma verdadeira epidemia mundial que atinge todas as faixas etárias, inclusive as crianças. Trata-se de uma doença crônica, definida como excesso de gordura corporal ${ }^{1}$.

Nos países em desenvolvimento, tem sido verificado um processo de transição nutricional nas últimas décadas, justificado pelo aumento progressivo da obesidade em substituição à desnutrição². No Brasil, comparando-se os dados do Estudo Nacional da despesa familiar, realizado em 1974/1975, com dados da pesquisa sobre padrões de vida realizada em $1996 / 1997$, somente nas regiões Sudeste e Nordeste, constatou-se aumento na prevalência da obesidade de $4,1 \%$ para $13,9 \%$ em crianças e adolescentes de 6 a 18 anos $^{3}$.

A prevalência de obesidade em crianças e adolescentes, as suas repercussões biopsicossociais, os altos índices de insucesso terapêutico no tratamento de obesidade na vida adulta e o grande risco da criança obesa se tornar um adulto obeso, fazem da obesidade um dos maiores problemas nutricionais na infân$\mathrm{Cia}^{4}$.

As causas na infância são de caráter multifatorial, envolvendo fatores bio- lógicos, psicológicos e ambientais ${ }^{5}$. O elevado consumo de alimentos gordurosos, com alta densidade energética, e a diminuição na prática de exercícios físicos são os dois principais fatores relacionados ao meio ambiente que colaboram para o excesso de gordura corporal ${ }^{6}$. Além disso, fatores como o equilíbrio entre consumo e acúmulo de gordura corporal, que podem ser geneticamente regulados, contribuem para a obesidade?

O padrão crescente de obesidade infantil pode levar a consequências negativas em curto e longo prazo. A longo prazo confere um risco aumentado para uma série de doenças, que podem ser divididas em seis grupos, a saber: doenças cardiovasculares (hipertensão, doença arterial coronariana e acidente vascular cerebral); desordens metabólicas (dislipidemias e diabetes mellitus); doenças respiratórias (apnéia do sono); certos tipos de câncer; calculose biliar; e doenças osteoarticulares ${ }^{8}$.

Na região Nordeste do Brasil, onde a desnutrição infantil outrora ganhava bastante destaque, os estudos sobre obesidade e sobrepeso são limitados a segmentos específicos e a distintas faixas etárias, além de utilizarem diferentes indicadores nutricionais, dificultando uma boa avaliação'. Portanto, tornou-se relevante investigar tal condição, pois o estudo poderá produzir melhor conhecimen- 
to da frequência de excesso de peso na faixa etária escolar (seis anos a dez anos incompletos) na referida região, o que pode retratar condições semelhantes àquelas encontradas em países em desenvolvimento, por ser uma das regiões de menor padrão econômico do país.

Desta maneira, o objetivo é descrever a prevalência de obesidade, sobrepeso, hábitos alimentares e a frequência de atividade física dos escolares da cidade de Campina Grande, Paraíba.

\section{MÉTODO}

Tratou-se de um estudo transversal e descritivo. O levantamento de dados ocorreu em quatro meses, de março a junho de 2008.

A amostra foi composta por 285 alunos matriculados do primeiro ao quinto ano do Ensino Fundamental, entre 6 e 10 anos. Foram excluídos os sujeitos com doenças crônicas que interferiam diretamente com o peso ou estatura e os que no momento da avaliação apresentaram algum impedimento para a obtenção de medidas antropométricas.

Para o cálculo da amostra foi utilizada a seguinte fórmula: $n=\left(Z^{2}\right.$. p.q. $\left.n\right)$ $/ \mathrm{d}^{2}(\mathrm{n}-1)+\mathrm{Z}^{2}$. p. $\mathrm{q}^{10}$, considerando um total de 24.543 escolares matriculados na rede pública e privada da zona urbana do município campinense ${ }^{11}$ e a prevalência de sobrepeso e obesidade de $20 \%{ }^{12}$. Adotou-se um nível de confiança de $5 \%$.

Com o intuito de respeitar a proporção entre o número de alunos de escolas da rede pública e privada, fizeram parte da pesquisa: quatro escolas da rede pública e duas da rede privada.

No primeiro momento, os pesquisadores fizeram uma visita a cada escola selecionada para uma etapa de esclarecimento à direção e aos professores, sobre os objetivos do estudo e os procedimentos que seriam realizados. Os pais e os alunos foram informados sobre a pesquisa através de uma carta circular.

Para obtenção dos dados foram utilizados um questionário com informações sobre o tipo de lanches e atividades físi- cas dos escolares e um protocolo para registro de informações, no qual foram registradas as medidas antropométricas, sexo, idade e tipo de escola.

Para aferição antropométrica cada criança foi medida e pesada por dois examinadores previamente treinados. Foi considerado como o valor da estatura e do peso, a média aritmética simples de duas mensurações.

Para obtenção do peso, foi utilizada uma balança digital da marca Camry, modelo EF221BW, com capacidade para $150 \mathrm{~kg}$ e precisão de $0,1 \mathrm{~kg}$. Os participantes se encontravam com roupas leves e foram posicionados na posição ortostática e com os pés juntos.

Para mensuração da estatura foi utilizado um estadiômetro com cursor e precisão de $0,1 \mathrm{~cm}$. Durante a aferição, os alunos foram orientados a retirar os sapatos e a permanecerem eretos; de costas para a régua; com os glúteos e tornozelos em contato com o estadiômetro; joelhos esticados e braços soltos ao lado do corpo. A cabeça dos alunos foi posicionada, considerando-se o Plano de Frankfurt (plano imaginário que passa pela órbita e meato auditivo externo). Foi solicitado aos mesmos que permanecessem em inspiração profunda durante a medição. Assim, o cursor foi colocado sobre a cabeça dos escolares, sendo a estatura correspondente ao valor imediatamente abaixo do cursor.

A partir das medidas supracitadas foi calculado o índice de massa corporal (IMC) por meio do quociente massa corporal/(estatura) ${ }^{2}$, sendo a massa corporal expressa em quilogramas $(\mathrm{kg})$ e a estatura em metros $(m)^{13}$.

Foram utilizadas as curvas e as tabelas de percentis do IMC do "Centers for Disease Control and Prevention" (CDC) para crianças e adolescentes (2 aos 20 anos de idade), de acordo com o gênero e a idade, (www.cdc.org/ growthcharts). Consideram-se com sobrepeso as crianças com IMC igual ou superior ao percentil 85 e inferior ao percentil 95 e, com obesidade, IMC igual ou superior ao percentil 95.

A avaliação da prevalência de obesidade e sobrepeso levou em considera- 
ção o gênero, faixa etária (grupo A de 6 anos completos a 8 anos e grupo $\mathrm{B}$ de 8 anos completos a 10 anos incompletos) e tipo de escola.

Pesquisou-se também, o tipo de lanche consumido pelos alunos na escola, bem como a quantidade de atividade física realizada. Para tal, foi enviado um questionário para os pais dos alunos responderem.

A atividade física foi quantificada em dias por semana, cada dia com duração média de 45 a 60 minutos de atividade, considerando em conjunto: aulas de educação física na escola e prática esportiva extracurricular. A atividade física foi classificada em quatro níveis: 0 dia/semana; $1 \mathrm{dia} /$ semana; 2 dias/semana; 3 ou mais dias/semana.

Os dados foram digitados em dupla entrada em um banco de dados eletrônico. Em seguida, foram organizados através de frequências absolutas e relativas. Para comparar as proporções entre a prevalência de obesidade/ sobrepeso entre os grupos de escolas particulares ou públicas, gênero e faixa etária foi utilizado o teste do quiquadrado. Os dados foram analisados com o programa Epi Info versão 3.3.4.
A análise estatística utilizou intervalo de confiança de $95 \%$.

A pesquisa foi aprovada pelo Comitê de Ética e Pesquisa do Hospital Universitário Alcides Carneiro (20081403004). Para autorização da participação dos alunos, os pais assinaram um Termo de Consentimento Livre e Esclarecido. O sigilo, o anonimato e a privacidade foram respeitados.

\section{RESULTADOS}

Conforme a Tabela 1, das 285 crianças estudadas, $141(49,5 \%)$ eram do sexo masculino. Com relação à idade, $47,7 \%$ tinham entre 6 e 8 anos (grupo A) e $52,3 \%$ tinham entre 8 e 10 anos (grupo B). No tocante à rede de ensino, $57(20 \%)$ escolares eram da rede privada e $228(80 \%)$ da pública.

$\mathrm{Na}$ Tabela 2, observa-se que a prevalência de sobrepeso encontrada foi de $14,7 \%$ e de obesidade $8,8 \%$, perfazendo uma prevalência de sobrepeso/obesidade igual a 23,5\%. Aproximadamente metade das crianças de escolas privadas apresentam-se com sobrepeso ou obesidade.

A Tabela 3 mostra que não houve diferença significativa de sobrepeso/obe-

Tabela 1: Distribuição da amostra em relação a faixa etária de acordo com o sexo e a rede de ensino, Campina Grande-PB, 2008

\begin{tabular}{lcccccccccc}
\hline \multirow{2}{*}{ Grupos } & \multicolumn{2}{c}{ Masculino } & \multicolumn{2}{c}{ Feminino } & \multicolumn{2}{c}{ Privada } & \multicolumn{2}{c}{ Pública } & \multicolumn{2}{c}{ Total } \\
& $\mathbf{n}$ & $\mathbf{\%}$ & $\mathbf{n}$ & $\mathbf{\%}$ & $\mathbf{n}$ & $\mathbf{\%}$ & $\mathbf{n}$ & $\mathbf{\%}$ & $\mathbf{n}$ & \% \\
\hline A (6 |- 8) & 65 & 46,1 & 71 & 49,3 & 29 & 50,9 & 107 & 46,9 & 136 & 47,7 \\
B (8 |- 10) & 76 & 53,9 & 73 & 50,7 & 28 & 49,1 & 121 & 53,1 & 149 & 52,3 \\
$\quad$ Total & 141 & 100 & 144 & 100 & 57 & 100 & 228 & 100 & 285 & 100 \\
\hline
\end{tabular}

Tabela 2: Distribuição do estado nutricional de acordo com o tipo de escola entre os escolares, Campina Grande-PB, 2008

\begin{tabular}{ccccrrr}
\hline Estado Nutricional & $\begin{array}{c}\text { Privada Pública } \\
\mathbf{n}\end{array}$ & $\mathbf{\%}$ & \multicolumn{1}{c}{ Total } & \multicolumn{1}{c}{$\mathbf{0}$} & $\mathbf{n}$ & \multicolumn{1}{c}{$\%$} \\
\hline Baixo peso & - & - & 10 & 4,4 & 10 & 3,5 \\
Eutrófico & 29 & 50,9 & 179 & 78,5 & 208 & 73,0 \\
Sobrepeso & 19 & 33,3 & 23 & 10,1 & 42 & 14,7 \\
Obesidade & 09 & 15,8 & 16 & 7,0 & 25 & 8,8 \\
Total & 57 & 100 & 228 & 100 & 285 & 100 \\
\hline
\end{tabular}


sidade (excesso de peso) entre os escolares de Campina Grande quando considerada a faixa etária. Entretanto, ao considerar o sexo, verifica-se maior prevalência em meninos ( $p<0,01$, Odds Ratio $=2,38$, IC: $1,34-4,22)$ e na rede de ensino privada $(p<0,01$, Odds Ratio $=4,67$, IC: $2,50-8,72$ ).

O excesso de peso foi mais frequente entre os alunos da rede privada $(49,1 \%)$ em relação aos alunos da rede pública $(17,1 \%)$, com risco 4,67 vezes maior entre os alunos das escolas particulares.
Pode-se observar na Tabela 4 que a frequência de escolares com três ou mais dias de atividade física foi de $33,7 \%$. 0 valor encontrado na rede privada foi $38 \%$ e na rede pública $28,9 \%$. Portanto, a maioria das crianças $(66,3 \%)$ pratica menos que três dias de atividade física por semana.

Na tabela 5 observa-se uma frequencia maior de lanches levados de casa para escola pelos alunos das escolas privadas $(83,7 \%)$.

Quando questionados quanto ao tipo de lanche preferido na escola (Tabe-

Tabela 3: Prevalência de sobrepeso/obesidade entre os escolares de acordo com sexo, faixa etária e rede de ensino, Campina Grande-PB, 2008

\begin{tabular}{|c|c|c|c|c|c|c|c|}
\hline \multirow[t]{2}{*}{$\begin{array}{c}\text { Variáveis } \\
\text { Independentes }\end{array}$} & \multicolumn{2}{|c|}{$\begin{array}{c}\text { Sobrepeso/ } \\
\text { Obesidade }\end{array}$} & \multicolumn{2}{|c|}{ Normal } & \multirow{2}{*}{$\begin{array}{c}\text { Qui-Quadrado } \\
\text { p }\end{array}$} & \multirow[t]{2}{*}{ Odds Ratio } & \multirow[t]{2}{*}{$\begin{array}{c}\text { Intervalo de } \\
\text { confiança }\end{array}$} \\
\hline & $\mathrm{n}$ & $\%$ & n & $\%$ & & & \\
\hline \multicolumn{8}{|l|}{ Sexo } \\
\hline Masculino & 44 & 31,2 & 97 & 68,8 & 0,0012662546 & 2,3864 & $1,34-4,22$ \\
\hline Feminino & 23 & 16 & 121 & 84 & & & \\
\hline \multicolumn{8}{|l|}{ Faixa etária } \\
\hline Grupo A & 30 & 22,1 & 106 & 77,9 & 0,2928528666 & & \\
\hline Grupo B & 37 & 24,8 & 112 & 75,2 & & & \\
\hline \multicolumn{8}{|l|}{ Rede } \\
\hline Privada & 28 & 49,1 & 29 & 50,9 & 0,0000009086 & 4,67900 & $2,50-8,72$ \\
\hline Pública & 39 & 17,1 & 189 & 82,9 & & & \\
\hline
\end{tabular}

Tabela 4: Prática de atividade física semanal entre os escolares, Campina GrandePB, 2008

\begin{tabular}{ccccccr}
\hline Dias/semana & \multicolumn{2}{c}{ Rede Privada } & \multicolumn{2}{c}{ Rede Pública } & \multicolumn{2}{c}{ Total } \\
& $\mathbf{n}$ & $\mathbf{\%}$ & $\mathbf{n}$ & $\mathbf{\%}$ & $\mathbf{n}$ & \multicolumn{1}{c}{$\%$} \\
\hline 0 & 01 & 2 & 02 & 4,4 & 03 & 3,1 \\
1 & 11 & 22 & 08 & 17,8 & 19 & 20,1 \\
2 & 19 & 38 & 22 & 48,9 & 41 & 43,1 \\
$\geq 3$ & 19 & 38 & 13 & 28,9 & 32 & 33,7 \\
Total & 50 & 100 & 45 & 100 & $95^{*}$ & 100 \\
\hline
\end{tabular}

* Número de questionários devolvidos pelos pais.

Tabela 5: Frequência de lanche levado de casa para escola, Campina Grande-PB, 2008

\begin{tabular}{|c|c|c|c|c|c|c|}
\hline & \multicolumn{2}{|c|}{ Rede Privada } & \multicolumn{2}{|c|}{ Rede Pública } & \multicolumn{2}{|c|}{ Total } \\
\hline & n & $\%$ & $\mathbf{n}$ & $\%$ & $\mathbf{n}$ & $\%$ \\
\hline $\begin{array}{l}\text { Sim } \\
\text { Não }\end{array}$ & $\begin{array}{l}48 \\
07\end{array}$ & $\begin{array}{l}87,3 \\
12,7\end{array}$ & $\begin{array}{l}32 \\
33\end{array}$ & $\begin{array}{l}49,2 \\
50,8\end{array}$ & $\begin{array}{l}80 \\
40\end{array}$ & $\begin{array}{l}66,7 \\
33,3\end{array}$ \\
\hline Total & 55 & 100 & 65 & 100 & $120 *$ & 100 \\
\hline
\end{tabular}

* Número de questionários devolvidos pelos pais. 
la 6), os escolares da rede privada apresentaram maior preferência pelo consumo de biscoito, refrigerante e salgadinho. Já os escolares da rede pública apresentaram maior consumo de biscoito, iogurte e salada de fruta.

Tabela 6: Frequência da preferência do consumo de lanche na escola, Campina Grande-PB, 2008

\begin{tabular}{lcc}
\hline Lanche & $\begin{array}{c}\text { Rede Privada } \\
\mathbf{( \% )}\end{array}$ & $\begin{array}{c}\text { Rede Pública } \\
\mathbf{( \% )}\end{array}$ \\
\hline Salgadinho & 50 & 34,9 \\
Sanduíche & 42 & 41,2 \\
Biscoito & 72 & 68,2 \\
Doces & 26 & 17,5 \\
Frutas & 32 & 52,4 \\
Salada de Frutas & 10 & 55,6 \\
Pipoca & 22 & 36,5 \\
Iogurte & 40 & 61,9 \\
Refrigerante & 54 & 42,8 \\
Suco Industrializado & 40 & 15,9 \\
Suco Natural & 46 & 42,9 \\
Leite Achocolatado & 22 & 54,0 \\
\hline
\end{tabular}

\section{DISCUSSÃO}

As prevalências de obesidade e sobrepeso entre escolares encontradas neste estudo foram semelhantes aos valores registrados em um estudo realizado na cidade de Recife-PE, onde a prevalência de sobrepeso foi $12,9 \%$ e de obesidade $8,2 \%$, totalizando $21,3 \%$ de excesso de peso ${ }^{12}$. Já em Natal-RN, as prevalências encontradas de sobrepeso e obesidade foram $11 \%$ e $22,6 \%$, respectivamente, sendo $33,6 \%$ de excesso de peso $^{14}$. Em Salvador-BA, a prevalência de obesidade foi $15,8 \%{ }^{15}$. Estudo anterior realizado entre os adolescentes de Campina Grande-PB identificou prevalências de obesidade e sobrepeso em adolescentes de $18,3 \%$ e $6,8 \%$, respectivamente, perfazendo uma prevalência de excesso de peso de $25,1 \%{ }^{16}$. Para comparação com dados de um estudo fora do nordeste, verificou-se em Cubatão-SP prevalência de $24 \%$ de excesso de peso entre escolares, sendo $13,64 \%$ com sobrepeso e $10,37 \%$ com obesidade ${ }^{17}$.

Como nesse estudo, a constatação de maior prevalência de sobrepeso/obesidade em crianças do sexo masculino também foi verificada em uma pesquisa ${ }^{18}$ na cidade de Florianópolis-SC e em outro estudo em São Paulo ${ }^{19}$. Entretanto, não há uma hipótese justificável para tais constatações, podendo apenas se tratar de casos isolados sem significância epidemiológica.

Apesar de não ter se avaliado as condições socioeconômicas, sabe-se empiricamente que, em países em desenvolvimento, estudantes da rede privada pertencem a uma classe de maior poder aquisitivo que os estudantes da rede pública. Portanto, os resultados obtidos podem indicar que escolares de nível socioeconômico mais elevado apresentam uma prevalência de excesso de peso maior que os da rede pública. Tal assertiva corrobora com resultados obtidos por estudos em outras cidades nordestinas, como Recife ${ }^{12}$, Natal ${ }^{14}$ e Salvador $^{15}$. Autores ${ }^{20}$ referiram que, na América Latina, a obesidade infantil é mais prevalente em famílias com nível socioeconômico mais elevado. Outro estudo ${ }^{21} \mathrm{com}$ crianças brasileiras menores de cinco anos encontrou maior prevalência de obesidade nas classes socioeconômicas elevadas do que nas classes baixas.

O padrão supracitado é característico dos países subdesenvolvidos, diferentemente do padrão de países desenvolvidos, nos quais, a obesidade infantil é mais prevalente na classe socioeconômica baixa, já que esta tem também acesso fácil a alimentos hipercalóricos e a classe mais elevada possui maior instrução acerca de padrões dietéticos e de atividade física ${ }^{22}$.

No Brasil, os hábitos alimentares proporcionados por uma renda familiar mais alta, com alimentos hipercalóricos e pobres em nutrientes, são relacionados a uma maior prevalência de sobrepeso/obesidade na faixa etária estudada ${ }^{15}$. Além disso, computadores e vídeo games são opções de lazer com peque- 
no gasto energético e mais acessíveis aos escolares de melhor nível socioeconômi$\mathrm{CO}^{23}$.

O achado da maioria das crianças praticar menos que três dias de atividade física por semana, pode ser justificado pela vida sedentária proporcionada por facilidades do mundo moderno. Embora já tenha sido demonstrada relação inversa entre prática esportiva sistemática e obesidade infantil, não foi encontrada menor atividade física entre escolares da rede privada, que possui maior prevalência de obesidade. Cabe questionar se a prática de exercício físico em escolares obesos da rede privada não seria uma tentativa dos pais de combater o distúrbio nutricional da criança, ou pelo maior esclarecimento sobre os benefícios da atividade física entre os pais da rede privada.

A constatação dos alunos da rede pública praticarem atividade física com menor frequencia pode estar relacionada com a carência que as escolas enfrentam em relação à disponibilidade de profissionais de educação física para orientar os alunos e quanto à inexistência de uma estrutura física adequada para a realização das atividades (quadras poliesportivas). Outros estudos, como uma pesquisa realizada em Brasília ${ }^{24}$ e outra em Salvador ${ }^{15}$ corroboram com a afirmação supracitada, evidenciando tal problemática.

Já nas escolas privadas parece não existir muitas dificuldades quanto à disponibilidade de recursos humanos ou materiais para realização de atividades físicas. Todavia, foram verificados no estudo altos índices de excesso de peso.

O fato dos alunos das escolas privadas levarem com maior frequência lanches de casa para a escola deve-se, provavelmente, a duas situações: a merenda escolar é oferecida diariamente em escolas da rede pública de Campina Grande, enquanto o lanche oferecido na rede privada é comercializado em cantina; além disso, os melhores índices socioeconômicos da criança da rede privada oferecem maior acesso a alimentos industrializados e com alto teor de gordura saturada ${ }^{25}$.
O lanche na escola tem sido alvo de grandes preocupações de pais e educadores, pois os mesmos, muitas vezes, não sabem o que servir as crianças nesse horário. As ofertas do mercado de alimento são muitas, porém, nem sempre são as mais adequadas para atender às necessidades nutricionais dos escolares ${ }^{26}$.

Apesar de a merenda ou lanche escolar representar apenas $15 \%$ da ingestão diária, controvérsias têm sido observadas em relação à sua composição, qualidade e quantidade. Mais do que representar um dos períodos para alimentação, a escola é responsável por uma parcela importante do conteúdo educativo global, inclusive do ponto de vista nutricional ${ }^{26}$. Diante da carência de informação e capacidade de dicernir o que é saudável ou não, os alunos acabam por desenvolver hábitos pouco sadios, os quais podem contribuir para o desenvolvimento de problemas de saúde, principalmente, em relação à obesidade infantil.

Nos últimos anos, tem sido cada vez mais intenso o debate internacional sobre o uso das cantinas escolares como espaço privilegiado de atuação na busca pela promoção da alimentação saudável. No Brasil, o Rio de Janeiro figura entre as cidades com iniciativas de regulamentação deste tipo de atividade. Em 2002, foi publicado o decreto municipal no 21.217 , que proíbe a comercialização dos seguintes alimentos nas escolas públicas municipais: bala, doce a base de goma, goma de mascar, pirulito, caramelo, refresco de pó industrializado e refrigerante. Esta medida foi bastante oportuna para a rede municipal de educação, tendo em vista que, do total de escolas à época ( $n=1033), 63 \%$ possuíam alguma forma de comercialização de refrigerantes, biscoitos, balas, doces e sorvetes e, em $25 \%$ das escolas com venda de alimentos, foi encontrada propaganda impressa de produtos industrializa$\operatorname{dos}^{27}$.

É importante salientar que dentre os alimentos proibidos no Rio de Janeiro em 2002 encontra-se o refrigerante, um dos produtos mais consumidos pelos participantes do presente estudo, tanto na 
rede pública como na particular, em detrimento de alimentos saudáveis como sucos e salada de frutas.

Portanto, diante da crescente prevalência de excesso de peso no nordeste brasileiro, as escolas, tanto públicas quanto particulares, não parecem vir cumprindo seu papel no estabelecimento de uma educação alimentar sadia para seus respectivos alunos.

O presente estudo verificou uma elevada prevalência de sobrepeso e obesidade entre os escolares da cidade de Campina Grande principalmente, entre os

\section{REFERÊNCIAS}

1. De Angelis RC. Riscos e prevenção da obesidade: fundamentos fisiológicos e nutricionais para o tratamento. Atheneu: São Paulo, 2003.

2. Batista Filho M, Rissin A. A transição nutricional no Brasil: tendências regionais e temporais. Cad. Saúde Pública. 2003; 19(Supl 1): 181-91.

3. Wang Y, Monteiro C, Popkin BM. Trends of obesity and underweight in older children and adolescents in the United States, Brazil, China, and Russia. Am J Clin Nutri. 2002; 75(6): 971-77.

4. Parizzi MR. Obesidade e outros distúrbios alimentares. 2 ed. São PauIo: Medsi; 2002.

5. Oliveira AM, Oliveira AC, Cerqueira EMM, Souza JS. Sobrepeso e obesidade infantil: influencias de fatores biológicos e ambientais em Feira de Santana/BA. Arq Bras Endocrinol Metab. 2003;47(2).

6. Dietz WH. Health consequences of obesity in youth: childhood predictors of adult disease. Pediatrics. $1998 ; 101(3): 518-25$.

7. Rosenbaum M, Leibel RL. The physiology of body weight regulation: relevance to the etiology of obesity in children. Pediatrics. 1997; 101(3): 525-39.

8. Rabelo LM. Fatores de risco para doença aterosclerótica na adolescência. J. Pediatr. 2001; 77(2):153-64. alunos de escolas privadas. Isto pode ser explicado pelo hábito alimentar não saudável, com ingestão frequente de alimentos ricos em carboidrato e gordura no lanche escolar, bem como pela alta frequência de sedentarismo; menos da metade realizavam atividade física três vezes na semana, o mínimo recomendado.

É notória a necessidade de implantação de políticas públicas voltadas para a prevenção da obesidade, bem como a intensificação da educação nutricional e do estímulo à atividade física no âmbito escolar.

9. Balaban G, Silva GAP, Motta MEFA. Prevalência de sobrepeso e obesidade em escolares de diferentes classes socioeconômicas em Recife, Pernambuco. Pediatria (São Paulo). 2001; 23(4): 285-9.

10. Rodrigues PC. Bioestatística. Niterói: Eduff; 1993.

11. Brasil. Ministério da Educação. Instituto Nacional de Estudos e Pesquisas Educacionais Anísio Texeira Resultado do Censo Escolar 2006. [on-line]. 2006. [citado 2007 maio 23]. Disponível em: www.inep.gov.br/basica/censo/Escolar/Matrícula/censoescolar_2006asp

12. Silva GAP, Balaban G, Motta MEF. Prevalência de sobrepeso e obesidade em crianças e adolescentes em diferentes condições socioeconômicas. Rev Bras Saúde Mat Inf. 2005; 5(1):53-9.

13. WHO. Obesity: Preventing and Managing the Global Epidemic. Report of a WHO Consultation on Obesity. WHO: Geneva; 1998.

14. Brasil LMP, Fisberg M, Maranhão HS. Excesso de peso de escolares em região do Nordeste Brasileiro: contraste entre as redes de ensino pública e privada. Rev. Bras. Saude Mater. Infant. 2007; 7(4):405-12.

15. Leão LSCS, Araújo LMB, Moraes LTLP, Assis AM. Prevalência de obesidade em escolares de Salvador, Bahia. Arq Bras Endocrinol Metabol. 2003; 47(2): 151-7. 
16. Nunes MMA, Figueiroa JN, Alves JGB. Excesso de peso, atividade física e hábitos alimentares entre adolescentes de diferentes classes econômicas em Campina Grande (PB). Rev. Assoc. Méd. Brás. 2007; 53(2):130-4.

17. Silva MCP, Ramos CHB, Costa RF. Estado nutricional e níveis pressóricos de escolares adolescentes da cidade de Cubatão-SP, Brasil. Rev. bras. crescimento desenvolv. hum. [online]. 2008. [citado 2010 maio 14];18(3): 288-97. Disponível em: http://pepsic.homolog.bvsalud.org/ scielo.php?script=sci_arttext\&pid=S0104$12822008000300008 \&$ lng $=p t \& n r m=$ iso

18. Soar C, Vasconcelos FAG, Assis MAA, Grosseman S, Luna MEP. Prevalência de sobrepeso e obesidade em escolares de uma escola pública de Florianópolis, Santa Catarina. Rev Bras Saúde Matern Infant. 2004; 4(4):391-7.

19. Simon VGN, Souza JMP, Leone C, Souza, SB. Prevalência de sobrepeso e obesidade em crianças de dois a seis anos matriculadas em escolas particulares no município de São Paulo. Rev. bras. crescimento desenvolv. hum. [on-line]. 2009. [citado 2010 maio 14]; 19(2). Disponível em: http://pepsic.homolog.bvsalud.org/ scielo.php?script=sci_arttext\&pid=S0104$12822009000200002 \&$ Ing =pt\&nrm $=$ iso.

20. Martorell R, Khan LK, Hughes ML, Grummer-Strawn LM. Obesity in Latin American women and children. $J$ Nutr. $1998 ; 128(9): 1464-73$.
21. Monteiro CA, Mondini L, Souza ALM, Popkin BM. Da desnutrição para a obesidade: a transição nutricional no Brasil. In: Monteiro CA. Velhos e novos males da saúde no Brasil: a evolução do país e de suas doenças. São Paulo: Hucitec; 1995.

22. Rolland-Cachera MF, Bellisle F. No correlation between adiposity and food intake: why are working class children fatter? Am J Clin Nutr. 1986; 44:779-87.

23. Silva MAM, Rivera IR, Ferraz MJRM, Pinheiro JT, Alves SWS, Moura AA, et al. Prevalência de fatores de risco cardiovascular em crianças e adolescentes da rede de ensino da cidade de Maceió. Arq Bras Cardiol. 2005;84(5):387-92.

24. Giugliano R, Carneiro EC. Fatores associados à obesidade em escolares. J. Pediatr. 2004; 80(1): 17-22.

25. Caldeira F. Consumo Alimentar em Presidente Prudente-SP: subsídios para políticas públicas. Dissertação (Mestrado em Geografia), Universidade Estadual Paulista Júlio de Mesquita Filho, UNESP; 2008.

26. Santana DA. A obesidade infantil nas escolas. [on-line]. [s. d.]. [citado 2010 maio 14]; Disponível em: http:/ /www.webartigos.com/articles/4718/ 1/a-obesidade-infantil-nas-escolas/ pagina1.html

27. Castro IRR. Promoção da alimentação saudável e da atividade física em nível municipal: a experiência da cidade do Rio de Janeiro. Revista $A B E-$ SO. $2003 ; 14$. 